\title{
Retrospective Study on Laparoscopic Management of Ectopic Pregnancy
}

\author{
Chaudhary Payal $\cdot$ Manchanda Rahul $\cdot$ Patil Vijay N.
}

Received: 9 March 2012/ Accepted: 23 September 2012/Published online: 10 November 2012

(C) Federation of Obstetric \& Gynecological Societies of India 2012

\begin{abstract}
Purpose To evaluate various laparoscopic methods for management of tubal ectopic pregnancy and study the incidence of ectopic pregnancy including the incidence of cornual ectopic pregnancy and conversion to laparotomy during laparoscopic procedure.

Methods A retrospective study was conducted in North Point Hospital, Delhi, on all laparoscopies conducted in 4 years, i.e., from January 2008 to December 2011.

Results Incidence of ectopic pregnancy was $4.62 \%$ (out of all laparoscopic surgeries over 4 years) and that for cornual pregnancy was $4.65 \%$ (out of all ectopic pregnancies); no laparotomy was done for the management of ectopic pregnancy. The site of ectopic pregnancy in the tubal pregnancy varied, with $76.75 \%$ in the ampullary region, $16.27 \%$ isthumic, $2.33 \%$ fimbrial, and $4.65 \%$ in the cornual region. Salpingectomy was done in $53.5 \%$ cases and $46.5 \%$ of patients underwent a conservative approach in the form of salpingostomy.

Conclusion The laparoscopic management of ectopic pregnancy is a safe and effective option with greatly reduced morbidity.
\end{abstract}

Chaudhary P., Fellow · Manchanda R. (₫),

Head of Department · Patil V. N., Fellow

Minimally Invasive Gynaecology Department, North Point

Hospital, 387, Panchsheel Park, New Delhi 110017, India

e-mail: drrahulmanchanda@rediffmail.com

Manchanda R., Head of Department

A-32, New Friends Colony, New Delhi 110025, India
Keywords Ectopic pregnancy - Cornual pregnancy · Laparoscopy

\section{Introduction}

Ectopic pregnancy is the implantation of the blastocyst outside the uterine cavity in an area which is not lined by the endometrium and uterine musculature; it is not conducive to further development and it not only leads to pregnancy loss, but also increases the incidence of maternal morbidity, mortality, and jeopardizes future conception. It is a common health problem in reproductive age group women, particularly in the first trimester of pregnancy and more so with the advent of ART and IVF, especially when performed for women with tubal factor infertility [1]. Most of them may be life threatening if not diagnosed, so it requires timely diagnosis and management. It accounts for $2 \%$ of first trimester pregnancies [2] - of them $98 \%$ are in various part of the fallopian tube and out of these, $70 \%$ are in the ampullary region, $12 \%$ isthmic, $11.1 \%$ fimbrial, $3.2 \%$ ovarian, $2.4 \%$ interstitial, and $1.3 \%$ in the abdominal cavity [3].

It can be managed medically, after proper selection of patients, with methotrexate which targets the trophoblastic tissue of the embryo. Surgical treatment is another option, especially in patients where medical treatment has failed or there is a contraindication to medical treatment and in ruptured ectopic cases.

Traditionally, Laparotomy has been the most commonly performed procedure, but since last decade, the laparoscopic 
approach is emerging as the gold standard for direct visualization of ectopic gestation, providing definitive diagnosis with simultaneous management with salpingostomy or salpingectomy, depending upon the clinical scenario. Not all ectopic pregnancies, however, are suitable for laparoscopic treatment. These include contraindication for laparoscopy, insufficient laparoscopic experience of the surgeon, or severe pelvic adhesion.

\section{Methods}

The present retrospective study was conducted at North Point Hospital, New Delhi. The data of all the cases of ectopic pregnancy managed at our center were collected from video recordings of all operative laparoscopy procedures including ectopic pregnancy. It is a retrospective, observational study of 929 laparoscopic procedures carried out from January 2008 to December 2011, over a 4-year period. 43 laparoscopic surgeries out of 929 laparoscopic procedures were done for ectopic pregnancies diagnosed by various means like history and examination, serial $\beta$-hcg estimations, and ultrasound. Nine were ruptured ectopic pregnancies out of the 43 cases and two were corneal ectopic pregnancies, both of which were unruptured. The surgeries were performed under general anesthesia. Salpingectomy was performed by stepwise desiccation at the mesosalpinx with bipolar electrocoagulation followed by cutting along the mesosalpinx using scissors. For salpingostomy, a linear incision was made on the most prominent and distended antimesenteric border of the fallopian tube with a monopolar electrode spatula, and products of conception were removed. Hemostasis was assured and any bleeding from the salpingostomy edges was managed with bipolar cautery, and the salpingostomy edges were left to heal by secondary intention.

\section{Results}

A total of 929 laparoscopic procedures were performed for various gynecologic indications, e.g., uterine fibroids, endometriosis, cyst of the ovaries, etc., and from these, 43 cases were done for unruptured and ruptured tubal ectopic pregnancies. The mean age of the women was 27.5 years and ranged between 23 and 35 years. The most common site of tubal ectopic pregnancy was the ampullary region in $33(76.75 \%)$ of cases, followed by the isthmic, cornual, and fimbrial regions. The detailed frequency of location of the tubal ectopic pregnancy is shown in Table 1.

In our study, the incidence of ectopic pregnancy was $4.62 \%$ out of the total laparoscopic surgeries, and that of cornual ectopic pregnancy from all ectopic pregnancies
Table 1 Frequency of sites of Tubal Ectopic pregnancy

\begin{tabular}{llc}
\hline Site of ectopic pregnancy & Total number & Percentage \\
\hline Ampullary & 33 & 76.75 \\
Cornual & 02 & 4.65 \\
Isthmic & 07 & 16.27 \\
Infundibular & 01 & 2.33
\end{tabular}

Total number of tubal ectopic pregnancy-43(n)

Table 2 Incidence and modes of surgical management of tubal ectopic pregnancy

\begin{tabular}{lll}
\hline Type of pregnancy or surgery & Total number & Percentage \\
\hline Ectopic pregnancy $(n)$ & 43 & $4.62^{N}$ (out of $N$ ) \\
Cornual pregnancy & 02 & 4.65 (out of $n$ ) \\
Laparotomy & 00 & 00 \\
Salpingectomy & 23 & 53.5 \\
Salpingostomy & 20 & 46.5 \\
\hline
\end{tabular}

$N$ (Total laparoscopic surgeries done)—929

was $4.65 \%$. Nine $(20.9 \%)$ were ruptured ectopic cases. The results of the surgical procedure are shown in the Table 2.

\section{Discussion}

The first ectopic pregnancy was diagnosed in the eleventh century. The first successful surgical intervention to treat an ectopic pregnancy was done by John Bard, a surgeon in New York, in 1759, and it was for the first time in 1883 that Robert Lawson Tait from Scotland introduced salpingectomy for the management of ruptured ectopic pregnancy. It was much later, in 1973, that Shapiro and Adler described treatment of ectopic pregnancy by laparoscopy. Since then, there has been tremendous progress in the field of laparoscopy, so much so that today, it is the standard surgical treatment of ectopic pregnancy. Laparoscopy is now preferable to laparotomy in the management of the unruptured ectopic pregnancy and even in case of a ruptured ectopic pregnancy in experienced hands. Patients managed laparoscopically have lesser blood loss, lesser postoperative pain with less need for analgesics, less postoperative adhesions, and early recovery vs laparotomy. The benefits of laparoscopy were illustrated in a systematic Cochrane database review of 35 randomized trials that compared laparoscopic salpingostomy with the open surgical approach [4]. Laparoscopic salpingostomy resulted in significantly shorter operation time ( 73 vs $88 \mathrm{~min}$ ), less perioperative blood loss (79 vs $195 \mathrm{ml}$ ), shorter duration of hospital stay (1-2 vs 3-5 days), shorter convalescence time 
(11 vs 24 days), and thus lower costs. Laparoscopic salpingostomy was found significantly less successful than the open surgical approach in the elimination of tubal ectopic pregnancy ( 2 RCTs, $n=165$, OR $0.28,95 \%$ confidence interval (CI) 0.09-0.86) due to a significantly higher persistent trophoblast rate in laparoscopic surgery (OR 3.5, $95 \%$ CI 1.1-11). However, the laparoscopic approach is significantly less costly than open surgery $(P=0.03)$ and long term follow-up $(n=127)$ showed no evidence of a difference in the intrauterine pregnancy rate (OR 1.2, $95 \%$ CI 0.59-2.5).

In general, the incidence of ectopic pregnancy is $2 \%$ of all pregnancies [2] and that of cornual ectopic pregnancy is around $2.4 \%$ [3] of all ectopic pregnancies. In our study, the incidence is more than usual because we have taken a different kind of study sample, i.e., the incidence out of all operative procedures done with laparoscopy instead of from all pregnancies.

Better availability of blood and blood components, volume expanders, optimal anesthesia care, and immediate conversion if necessary to laparotomy dared the gynecologist to perform the laparoscopy safely and save the fallopian tube in a large percent of cases. In all our cases, including the ruptured ectopic cases, the hemoperitoneum was managed by suction with a 5-mm suction cannula. In our cases, hemoperitoneum ranged from 1,000 to $1,700 \mathrm{ml}$ in most of the cases, except in two cases it was 2,500-3,000 ml. But, in none of the cases did the hemoperitoneum appear to be a deterrent in surgery, and patients were managed successfully intraoperatively and postoperatively. In cases in which salpingostomy was performed, products were removed with hydrodissection, and later hemostasis was achieved with bipolar coagulation. The decision to proceed with salpingectomy was done in cases where bleeding continued even after applying various hemostatic methods, the fallopian tube was damaged irreparably, or the patient had completed her family. Some authors use vasopressin as a $20 \mathrm{IU} / 20 \mathrm{ml}$ normal saline injection instilled in the periphery of the gestation sac in the mesosalphinx, uterine, or tubal musculature to reduce the risk of hemorrhage [5]. But, we did not use it in any of our cases.

In cornual pregnancy, surgical treatment usually requires laparotomy. But, with the introduction of laparoscopic surgery, it is now possible to manage these cases laparoscopically. There are successful case reports of laparoscopic resection of cornual pregnancies [5]. In our study, both cornual pregnancies which were approximately $3 \mathrm{~cm}$ in size were managed by salpingectomy. In a study by MacRae et al. [5] on 11 cases of cornual ectopic pregnancy, cornual resection was done in $30 \%$ and cornuostomy in $70 \%$ of cases with a $9.09 \%$ conversion to laparotomy, thus showing that it can be managed both by Salpingectomy and salpingostomy. In both our cases, diagnosis was made with the help of ultrasound, but location could not be ascertained as cornual. The literature shows that early preoperative identification of a cornual ectopic pregnancy remains difficult [6] and most of the cases can be identified only intraoperatively.

In our study, all 43 cases were managed laparoscopically and laparotomy was not required in any of these cases for any reason like hemorrhage or other intraoperative complications, and it was possible to conserve the tube in $46.5 \%$ of cases. Hemorrhage is a key factor requiring conversion from laparoscopy to laparotomy [6]. The decision for timely salpingectomy with proper use of the bipolar energy to control bleeding is a key factor and a lot depends on the operating surgeon's experience. In our study, the decision for salpingectomy was taken intraoperatively. Other factors that contribute to the salpingectomy are recurrent ectopic pregnancy in the same tube, a severely damaged tube, a large tubal pregnancy of $\geq 5 \mathrm{~cm}$, and women who have completed their family [7]. Massive hemoperitoneum is not a contraindication for laparoscopic management of ectopic pregnancy. Postoperatively, the patient can be discharged within $24 \mathrm{~h}$. With adequate experience in laparoscopy, i.e., with an experienced laparoscopic surgeon [5] and with proper instruments, most if not all of the patients with ectopic pregnancy can be treated successfully by laparoscopy, whatever the gestation size or location, the number of gestations, or the presence of tubal rupture.

In a review by Tulandi et al. [7], laparoscopic salpingostomy remains the definitive and universal treatment of ectopic pregnancy in patients who are hemodynamically stable and who wish to preserve their fertility. The reproductive performance after salpingostomy appears to be equivalent to or better than salpingectomy, but the recurrent ectopic pregnancy rate may be slightly greater. Methotrexate is an alternative to surgical treatment in selected patients who fulfill strict inclusion criteria, including compliance with follow-up evaluation. Interstitial pregnancy also can be treated with methotrexate. Otherwise, a cornual resection or salpingotomy can be done. Although, it is feasible by laparoscopy, the laparoscopic approach should be done only by those who have an expertise in laparoscopic suturing.

\section{Conclusion}

Laparoscopy is a safe and effective procedure in experienced hands with minimal morbidity to the patient, even in cases of cornual pregnancy and ruptured ectopic pregnancy. During laparoscopy, direct visualization not only of the pathological fallopian tube, but also of the normal tube 
as well as rest of the abdominal cavity provides a more panoramic view and may help in future conception management.

\section{References}

1. Strandell A, Thorburn J, Hamberger L. Risk factors for ectopic pregnancy in assisted reproduction. Fertil Steril. 1999;71:282.

2. Centers for Disease Control and Prevention (CDC). Ectopic pregnancy-United States, 1990-1992. MMWR Morb Mortal Wkly Rep 1995;44:46.
3. Bouyer J, Coste J, Fernandez H, et al. Sites of ectopic pregnancy: a 10 year population-based study of 1800 cases. Hum Reprod. 2002; 17:3224.

4. Hajenius PJ, Mol F, Mol BW, et al. Interventions for tubal ectopic pregnancy. Cochrane Database Syst Rev 2007; CD000324.

5. MacRae R, Olowu O, Rizzuto MI, et al. Diagnosis and laparoscopic management of 11 consecutive cases of cornual ectopic pregnancy. Arch Gynecol Obstet. 2009;280:59-64.

6. Walid MS, Heaton RL. Diagnosis and laparoscopic treatment of cornual ectopic pregnancy. Ger Med Sci. 2010;8:16.

7. Tulandi T, Saleh A. Surgical management of ectopic pregnancy: clinical obstetrics and gynecology. 1999;42-1:31-8. 